Julie LeBlanc, Genèses de soi. L'écriture du sujet féminin dans quelques journaux d'écrivaines, 2008

\title{
Catherine Viollet
}

\section{(2) OpenEdition}

1 Journals

\section{Édition électronique}

URL : http://journals.openedition.org/genesis/571

DOI : $10.4000 /$ genesis.571

ISSN : 2268-1590

Éditeur :

Presses universitaires de Paris Sorbonne (PUPS), Société internationale de génétique artistique littéraire et scientifique (SIGALES)

Édition imprimée

Date de publication : 1 janvier 2011

Pagination : 230-232

ISBN : 978-2-84050-749-9

ISSN : 1167-5101

\section{Référence électronique}


« peuple » à travers le processus de rédaction du document «Église et communauté nationale » (1981), l'un des documents de l'épiscopat argentin les plus influents sur un plan politique, publié dans les dernières années de la dictature militaire. La rédaction de ce document a été confiée à un groupe d'acteurs appartenant au Movimento de Sacerdotes para el Tercer Mundo (MSTM) (Mouvement des prêtres pour le tiers-monde), mouvement qui a surgi et s'est développé en Argentine entre 1968 et 1973, qui a cherché à concilier le concile Vatican II et l'action politique sur un plan à la fois théologique et pastoral. L'analyse de ce dossier, composé de quatre brouillons dactylographiés portant des corrections autographes, permet à Juan Eduardo Bonin de mesurer l'effort que déploient les rédacteurs pour traduire les contenus idéologiques du secteur du catholicisme qu'ils incarnent dans les termes plus généraux du dispositif discursif de la Conférence épiscopale argentine (CEA). La comparaison entre le dernier dactylogramme et le texte publié montre néanmoins à quel point les évêques ont surveillé cette opération.

Dans une approche plus classique en génétique textuelle, "La fenêtre éclairée" : uma escrita constelar » de Carla Cavalcanti e Silva tente de saisir la dynamique compositionnelle à l'œuvre dans les brouillons de Proust à travers l'analyse du mouvement d'écriture qui, d'une première ébauche conduit à la rédaction définitive de cet épisode bien connu de $L a$ Prisonnière. En suivant la progression de ce mouvement à travers l'étude des cahiers 53 et 55, notamment, Carla Cavalcanti e Silva souligne d'abord l'absence de linéarité dans l'usage que Proust fait du support cahier : utilisation discontinue des rectos, puis des versos, avec aménagement de blancs remployés lors d'étapes ultérieures, détachement et déplacement des feuillets, témoignant d'un mouvement d'aller et retour incessant entre les deux cahiers. À cet usage non linéaire du support répond une écriture qui, elle aussi, progresse de façon discon- tinue : concentration puis éclatement des contenus ou signifiés thématiques, déplacement et dissémination des motifs, traitement en parallèle des connotations euphorique ou dysphorique qui leur sont associées, puis fusion ou tressage dans la construction d'ambivalences, bref, tout un ensemble complexe d'opérations que Carla Cavalcanti e Silva porte à la lumière et qui la conduisent à parler de l'écriture proustienne comme d'une écriture en constellation.

« Uma pequena esfera iridescente » (Une petite sphère iridescente) est un hommage très personnel que rend Claudia Amigo Pino à son maître et ambassadeur de la critique génétique au Brésil, le Prof. Philippe Willemart. Présenté lors du lancement du livre Processos de criação 4 (Processus de création), ce texte est l'occasion pour Claudia Amigo Pino de retracer sa rencontre avec une pensée qui se poursuit inlassablement à contre-courant de la doxa universitaire et qui, animée par une grande ambition universaliste, opère par coups de sonde portés sur des champs aussi éloignés que la physique et la psychanalyse, la biologie et la philosophie, en passant par les mathématiques et la théorie littéraire pour s'affronter aux questions que suscite l'approche des manuscrits d'écrivain : qu'est-ce qu'écrire? comment écrit-on? en quoi les processus d'écriture nous éclairent-ils sur la manière qu'a l'humain de penser et d'ordonner le monde qui l'entoure ? Le commentaire va au-delà du compte rendu d'ouvrage classique pour constituer une invitation enthousiaste à la découverte de l'ensemble de la (très abondante) production de Philippe Willemart.

Le numéro se clôt sur une visite en éclaireur de l'atelier de Pedro Nava par Edina Regina P. Panichi dont se réjouiront sûrement les généticiens souhaitant se lancer dans l'étude des manuscrits de cet écrivain décédé en 1984, à une époque où la critique génétique ébauchait à peine ses premiers pas au Brésil.
Julie LeBlanc, Genèses de soi. L'écriture du sujet féminin dans quelques journaux d'écrivaines, Montréal, Éditions du remue-ménage, 2008, 238 p.

\section{Compte rendu par Catherine Viollet}

Genèses de soi, de Julie LeBlanc (professeur à l'université de Toronto, Canada), relève, de manière stimulante et novatrice, un double défi : appliquer la génétique textuelle à l'écriture diaristique - démarche qui peut sembler paradoxale au premier abord ; et l'appliquer à un corpus d'auteurs francophones de sexe féminin - fait suffisamment exceptionnel, dans la critique génétique de langue française ${ }^{5}$, pour qu'il mérite d'être souligné.

Si le titre choisi évoque l'entreprise, commune aux différents auteurs, d'autoengendrement, de construction du sujet dans et par l'écriture, les cas étudiés abordent des configurations fort diverses : journal de voyage, journal de genèse, journal palimpseste, roman-journal... S'ils présentent des écarts par rapport au genre canonique, tous possèdent une dimension génétique - des phases de réécriture et de métamorphose ; ils ont aussi en commun des préoccupations esthétiques, et le souci d'exprimer un point de vue féminin, voire féministe.

Une première partie, intitulée « Genèse d'un journal », réunit deux études qui ont pour même objet la transformation d'un journal dont une version a été diffusée oralement sur Radio-Canada, puis remaniée pour être publiée.

Journal intime, de Madeleine Ouellette-Michalska, radiodiffusé à partir de l'été 1982, se compose de nombreux cahiers (déposés à la Bibliothèque natio-

4. São Paulo, Perspectiva, 2009.

5. Il suffit, pour s'en convaincre, de feuilleter la «Bibliographie : études génétiques, éditions, manuscrits » de l'un des derniers numéros de la revue Genesis, recensant les publications dans ce domaine de janvier 2008 à janvier 2010 : moins de $4 \%$ des publications citées concernent des auteurs de sexe féminin. Voir Genesis, $n^{\circ} 30$, « Théorie : état des lieux », p. 285-295. 
nale du Québec à Montréal) ; La Tentation de dire. Journal (Québec Amérique, 1985) est une version remaniée et augmentée du texte initialement diffusé. Les écarts entre les deux versions de ce journal sont étroitement liés à une pratique intensive d'intertextualité, d'entrelacement et de tissage, incluant notamment des extraits du journal de la grand-mère de l'auteur (déjà largement enchâssés dans Journal intime), d'un roman en cours de rédaction (La Maison Trestler), de récits de voyage... Il importe à Julie LeBlanc de montrer les enjeux textuels de cette évolution, vers cette architexture hybride où différentes voix se " chevauchent», contestant une "vision restreinte et univoque du sujet », et correspondant au souci de mettre en valeur l'importance des composantes intersubjectives et interdiscursives de l'identité féminine.

Tout à fait contemporains, les deux journaux de Nicole Brossard dont il est ensuite question - l'un radiodiffusé en août 1983, l'autre publié un an plus tard sous le titre Journal intime ou Voilà donc un manuscrit (Herbes Rouges, 1984) se situent à peu près dans la même relation, le premier constituant l'avant-texte du second. Outre les remaniements stylistiques ponctuels du journal radiodiffusé, s'ajoutent alors de nouvelles strates énonciatives, tels que poèmes « accueillant l'expression du vécu » (Nicole Brossard), « postures », récits et clausules. Mettant en scène une situation interlocutive, l'écriture diaristique affirme sa vocation dialogique : elle cherche à « créer un nouvel espace littéraire », démystifiant les aspects androcentriques du genre, repensant les relations entre vie et écriture, et légitimant « la subjectivité singulière et collective des femmes » (N. Brossard).

Très riche sur le plan de l'analyse génétique, la deuxième partie est centrée sur les journaux accompagnant la genèse d'une œuvre : les carnets d'écriture de Marie-Claire Blais, un journal d'écriture d'Annie Ernaux.

Les carnets de Marie-Claire Blais, qu'elle appelle notebooks, concernent les phases préparatoires de la rédaction d'un roman, Une saison dans la vie d'Emmanuel (Éditions du Jour, 1965 ; prix Médicis 1966), et d'une nouvelle, « Testament de Jean-le-Maigre à ses frères » (Liberté, vol. XIV, $\left.\mathrm{n}^{\circ} 3,1972\right)^{6}$. Spectaculaires sur le plan graphique en raison de nombreux dessins au crayon et à l'aquarelle qui «participent de la genèse », ces carnets sont riches de notes de lecture préparatoires, et de réflexions métatextuelles soigneusement datées sur l'œuvre à venir : profil diégétique, schéma narratif, cadre spatio-temporel, caractéristiques des personnages, conditions d'intelligibilité, présupposés de l'organisation narrative, stratégies textuelles, fragments de scénarios auxquels se mêlent des notations tirées du vécu de l'auteur et de ses diverses activités contemporaines de la rédaction. Ces carnets, " espaces d'invention » au contenu hétérogène et multidimensionnel, permettent au généticien de retracer la diachronie de l'activité créatrice à travers ses hésitations, ses virtualités et ses choix.

L'étude génétique de Passion simple (Gallimard, 1991) d'Annie Ernaux s'appuie sur un matériau aussi riche que complexe : d'une part, un autre texte publié, $S e$ perdre (Gallimard, 2001); d'autre part, un journal d'écriture inédit et des centaines de feuillets qui composent le dossier de genèse de ce récit (fin 1989-début 1991). Les deux textes publiés se font écho dans une relation spéculaire : si Passion simple est le court récit d'une passion amoureuse et charnelle vécue par l'auteur-narratrice avec un diplomate russe - une " passion qui ressemblait à l'écriture d'un livre ${ }^{7} »-$, Se perdre est le journal intime qu'a tenu Annie Ernaux durant la période relatée (et sur lequel s'est appuyée la genèse de Passion simple $)^{8}$; il a vis-à-vis de ce récit, précise Annie Ernaux, le statut d' " autodossier ${ }^{~} »$, de document endogénétique.

Quant au journal d'écriture qui précède et accompagne la rédaction de Passion simple, rédigé sur des feuilles volantes, il comporte diverses étapes de l'élaboration du récit, des listes de mots aux notes de régie, des scénarios ordonnant les événements à la recherche de la juste distance énonciative... Il abrite ce « dialogue interne à l'écriture », ses virtualités, ses incertitudes, ses stratégies, ses évaluations, ses réflexions quant à la censure sociale du discours érotique féminin. Les fonctions exploratoires du journal d'écriture portent aussi, comme le souligne Julie LeBlanc, sur les choix esthétiques : s'agira-t-il d'un témoignage, de confidences, d'un manifeste, d'un procèsverbal ? Quelle distance instaurer entre le moi acteur et le moi narrateur? L'analyse de cinq feuillets extraits de l'abondant dossier manuscrit de Passion simple met en lumière le processus de dépersonnalisation, d'objectivation et d'universalisation (p. 131) caractéristique de l'œuvre d'Ernaux.

Ce dossier exceptionnel est l'occasion, pour Julie LeBlanc, de montrer comment, entre « complexité et complicité », se complètent et se démultiplient trois pratiques d'écriture de nature différente : spéculative (journal d'écriture), descriptive (Passion simple), et diaristique (Se perdre).

La dernière partie de l'ouvrage comprend un chapitre de réflexions théoriques sur la relation entre fiction et non-fiction, et sur les critères susceptibles - ou non de les différencier (intention illocutoire, situation d'énonciation et valeur référentielle des déictiques, onomastique, statut diégétique de l'auteur et du narrateur, ancrage référentiel, informations para- et extratextuelles...).

6. Sur les carnets de genèse du « Testament de Jean-le-Maigre », voir aussi Julie LeBlanc, «Marie-Claire Blais et ses journaux d'écriture », Métamorphoses du Journal. De Rétif de la Bretonne à Sophie Calle, dir. C. Viollet et M.-F. Lemonnier-Delpy, Louvain-la-Neuve, Academia-Bruylant, 2006, p. 149-169.

7. Journal inédit de Passion simple, cité par Julie LeBlanc, p. 114

8. Voir Philippe Gasparini, «Annie Ernaux. De Se perdre à Passion simple », Genèse et autofiction, dir. J.-L. Jeannelle et C. Viollet, Louvainla-Neuve, Academia-Bruylant, 2007, p. 149174.

9. Annie Ernaux, L'Écriture comme un couteau. Entretien avec Frédéric-Yves Jeannet, Paris, Stock, 2003. 
Dans le prolongement de ces réflexions, Julie LeBlanc examine le dossier génétique (documents préparatoires, différents états manuscrits) d'un roman autobiographique de Madeleine Monette, intitulé Le Double suspect (Quinze, 1988). Imitant le discours autobiographique, cette fiction entrecroise dans une structure d'emboîtement trois journaux fictifs, créés de toutes pièces (le second réécrivant le premier, le troisième commentant cette opération et ses implications), et met en scène un personnage-écrivain «exhibant le dispositif de production » de son œuvre et les différentes phases de la genèse. Ce montage d'un triple simulacre d'écriture diaristique met en lumière le travail d'interaction, d'assimilation et de transformation textuelle, tandis que la construction interdiscursive et discontinue rend compte - dans une perspective féministe - de l'incessante interaction entre image de soi et image de l'Autre.

Au fil de ce parcours se confirme une double démonstration : de ce que le journal, « au cœur de la poeisis littéraire », peut apporter à la critique génétique; et de ce en quoi cette dernière enrichit l'étude des journaux.

L'ouvrage s'appuie sur une bibliographie solide, la description des fonds d'archives et la reproduction de certains manuscrits. On regrette cependant l'inconfort de lecture dû au fait que l'appareil de notes est renvoyé en fin de volume, et que les transcriptions de manuscrits ne respectent pas les normes en vigueur; et surtout, on regrette que l'auteur ait dû renoncer, faute de place, à l'analyse détaillée des réécritures. 